\title{
ESTUDO DE TRANCAS COM SISTEMA DIGITAL DE MONITORAMENTO
}

\section{Renan Mendes de Almeida ${ }^{1}$ \\ Thiago Miranda $\mathrm{Cruz}^{2}$ \\ Renan Gonçalves ${ }^{3}$ \\ Luiz Paulo Rosa ${ }^{4}$ \\ Ronaldo Emerick Moreira ${ }^{5}$ \\ Carlos Alberto Segato ${ }^{6}$}

Resumo: Nós nunca estamos completamente seguros em nossos ambientes frequentados no cotidiano. Acessos indevidos de pessoas em nossos meios, como casas e empresas são possíveis e em muitos casos realizados com muita facilidade, em especial as nossas residências. Não menos importante é a nossa segurança na rede, pois podemos ter informações importantes violadas. Tendo em mente o problema em questão, o monitor de acessos traz consigo a união em termos materiais e digitais, tornando mais seguro o acesso aos ambientes em que for instalado, oferecendo informações em páginas da web que podem ser monitoradas de qualquer lugar.

Palavras-chave: Segurança; Informação; Zeladoria; Acesso; Tecnologia.

\footnotetext{
1 Ciência da Computação/Faculdade Anhanguera de São José dos Campos, Brasil. E-mail: renanalmeidahistory@gmail.com.

2 Ciência da Computação/Faculdade Anhanguera de São José dos Campos, Brasil. E-mail: casttagnacci@gmail.com.

${ }^{3}$ Ciência da Computação/Faculdade Anhanguera de São José dos Campos, Brasil. E-mail: grenan04@gmail.com. 4 Ciência da Computação/Faculdade Anhanguera de São José dos Campos, Brasil. E-mail: luizpauloanhanguera@gmail.com.

5 Ciência da Computação/Faculdade Anhanguera de São José dos Campos, Brasil. E-mail: ronaldo_emerick@anhanguera.com.

6 Ciência da Computação/Faculdade Anhanguera de São José dos Campos, Brasil. E-mail: carlos.segato@anhanguera.com.
} 